\title{
Blunt anal sphincter injury related to pelvic bone fracture
}

Received January 10, 2020

Revised May 19, 2020

Accepted May 20, 2020

Correspondence to

Pil Young Jung

Department of Surgery, Yonsei

University Wonju College of Medicine,

20 Ilsan-ro, Wonju 26426, Korea

Tel: +82-33-741-0882

Fax: +82-33-741-0574

E-mail: surgery4trauma@yonsei.ac.kr

\author{
Sanghyun An, Hye Youn Kwon, Pil Young Jung \\ Department of Surgery, Yonsei University, Wonju College of Medicine, Wonju, Korea
}

\begin{abstract}
There have been limited data that can guide the management of patients with traumatic anal sphincter injury, particularly in civilian settings. Therefore, treatment should be applied on a case-by-case basis. Here we present the case of a 32-year-old man who presented with blunt anal sphincter injury. In this study, we discuss the management of anal sphincter injury and posterior urethral injury.
\end{abstract}

Key Words: Anal sphincter, Trauma, Colostomy

\section{Introduction}

The standard management of anal injury has been developed primarily based on damage in military settings. However, because anal damage occurs through different mechanisms in civilian settings, its treatment also varies. It is important to minimize infectious complications and preserve anal sphincter function in the treatment of anal injury. In this report, we present a case of anal sphincter injury in the civilian setting and discuss treatment options.

\section{Case presentation}

A 32-year-old man who had no significant medical history visited our trauma center with an injury caused by a 2-m fall. The exact mechanism of the injury was not confirmed, but an external wound was observed at the 7 oclock position around the anus (Fig. 1). The patient's vital signs were stable. Initial imaging studies showed pelvic ring injury-both superior and inferior ramus fractures. In addition, computed tomography revealed multiple air bubbles around the anus, indicating anorectal injury (Fig. 2). Urethral catheterization was unsuccessful and the patient presented with severe pain, indicating urethral injury. Surgery was performed for wound exploration, and severe anal sphincter injury and hematoma of the bladder were observed (Fig. 3). Upon intraoperative sigmoidoscopy, we confirmed that the rectum and sigmoid colon were not injured. The anal wound was cleaned and irrigated, and two silicone drains were inserted (Fig. 4). Fecal diversion with loop sigmoid colostomy was performed. After

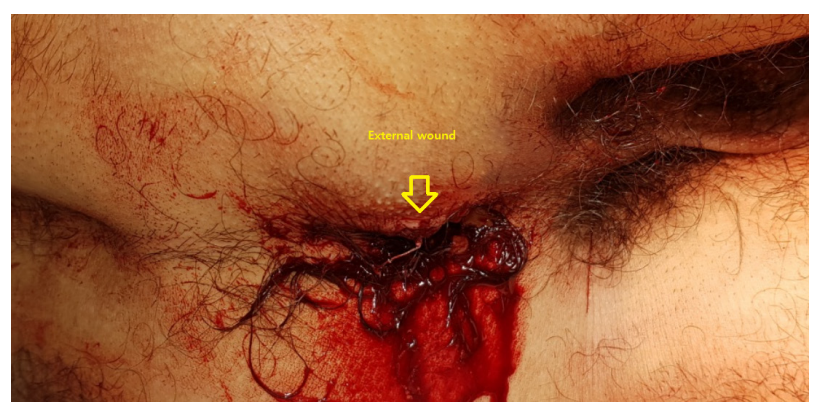

Fig. 1. External wound, anus.

(c)This is an Open Access article distributed under the terms of the Creative Commons Attribution Non-Commercial License (http://creativecommons.org/licenses/by-nc/4.0) which permits unrestricted noncommercial use, distribution, and reproduction in any medium, provided the original work is properly cited.

Copyright (c) 2020 Korean Association for Research, Procedures and Education on Trauma. All rights reserved. 


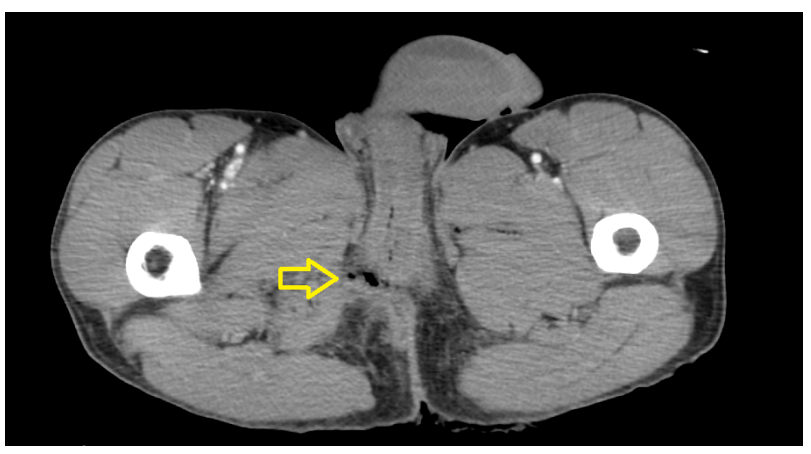

Fig. 2. Initial computed tomography scan showing multiple air bubbles around the anus and perineum.

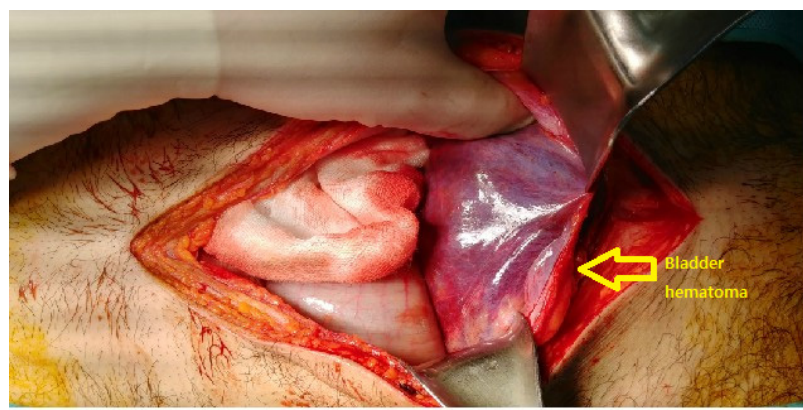

Fig. 3. Intraoperative finding: hematoma of the bladder.

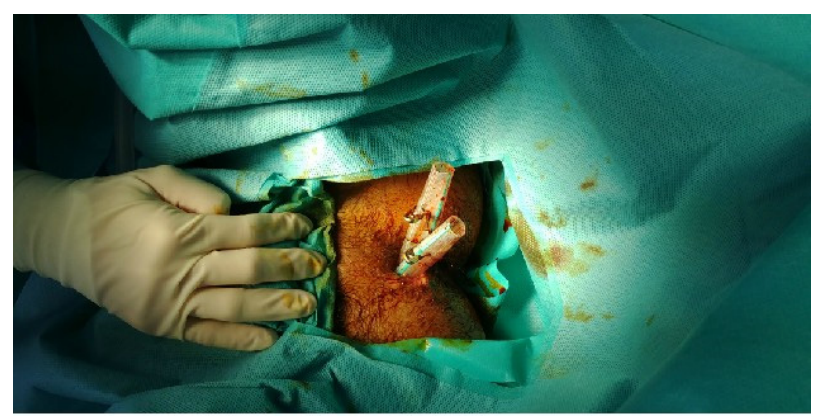

Fig. 4. Postoperative findings.

suprapubic cystostomy was performed by a urologist, endoscopic primary realignment was attempted, but the procedure failed. After surgery, magnetic resonance imaging was performed for further evaluation of sphincter damage, and we confirmed that the internal and external anal sphincters were damaged on the right side by approximately $1.2 \mathrm{~cm}$ (Fig. 5). The patient underwent closed reduction and screw fixation for the pelvic ring injury on the $13^{\text {th }}$ day. The silicone drains were removed on the $26^{\text {th }}$ day, and no wound infection was observed (Fig. 6). The patient was discharged on the $30^{\text {th }}$ day after suprapubic cystosto-

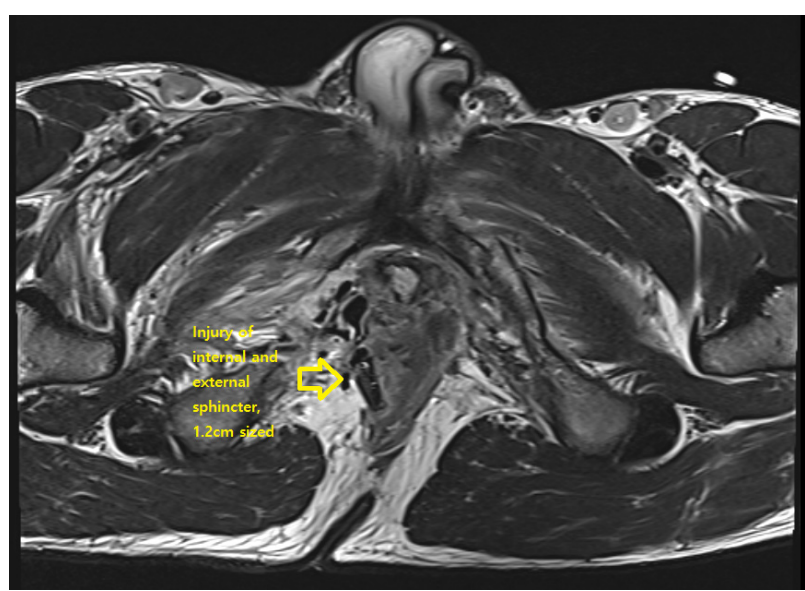

Fig. 5. Magnetic resonance imaging findings.

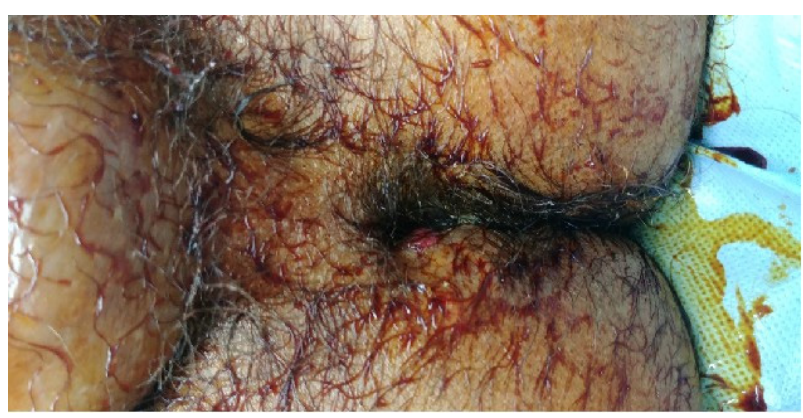

Fig. 6. Wound on the $26^{\text {th }}$ day.

my and colostomy.

\section{Discussion}

The principles of anorectal trauma management include the "4 Ds": diversion, distal rectal washout, drainage of the presacral area, and direct repair of the injury (1). However, these principles are mainly applicable to penetrating trauma in military settings, and data regarding anorectal injury in civilian settings are still lacking. Therefore, treatment should be applied on a case-by-case basis. In the present study, we performed fecal diversion with loop sigmoid colostomy because we determined that the patient had severe anal sphincter injury and was at a high risk of wound infection. We are currently planning colostomy reversal and sphincter reconstruction, but it is difficult to predict whether the anal sphincter function will be restored and, if so, to what extent. To predict the functional outcome, anorectal manometry can be performed prior to colostomy reversal (2). 
In men, blunt pelvic trauma with pelvic ring injury causes posterior urethral injury (PUI) in up to $10 \%$ of patients, and long-term morbidity due to PUIs is associated with urethral stricture, erectile dysfunction, and urinary incontinence $(3,4)$. In our patient, we performed suprapubic cystostomy because urethral catheterization failed. Primary realignment also failed as a result of the severe injury; therefore, a suprapubic catheter was inserted, and delayed urethroplasty is being currently planned. Clinicians should monitor complications and functional recovery after anal sphincter reconstruction and urethroplasty.

\section{Conflict of interest}

No potential conflict of interest relevant to this article was reported.

\section{References}

1. Ahern DP, Kelly ME, Courtney D, Rausa E, Winter DC. The management of penetrating rectal and anal trauma: a systematic review. Injury. 2017;48(6):11338.

2. Lee L, McKendy KM. Management of trauma to the rectum and anus. Dis Colon Rectum. 2018;61(11): 1245-8.

3. Bjurlin MA, Fantus RJ, Mellett MM, Goble SM. Genitourinary injuries in pelvic fracture morbidity and mortality using the National Trauma Data Bank. J Trauma Acute Care Surg. 2009;67(5):1033-9.

4. Elshout PJ, Veskimae E, MacLennan S, Yuan Y, Lumen N, Gonsalves M, et al. Outcomes of early endoscopic realignment versus suprapubic cystostomy and delayed urethroplasty for pelvic fracture-related posterior urethral injuries: a systematic review. Eur Urol Focus. 2017;3(6):545-53. 